\title{
Significance of target cell infection and natural killer cells in the anti-tumor effects of bacillus Calmette-Guerin in murine bladder cancer
}

\author{
TEPPEI SONODA, KAZUNOBU SUGIMURA, SHIN-ICHI IKEMOTO, \\ HIDENORI KAWASHIMA and TATSUYA NAKATANI
}

Department of Urology, Osaka City University Graduate School of Medicine, Osaka 545-8585, Japan

Received February 2, 2007; Accepted March 12, 2007

\begin{abstract}
Although intravesical instillation of bacillus Calmette-Guerin (BCG) is a clinically well-recognized therapy for bladder carcinoma in situ and recurrence prophylaxis, these mechanisms have not been fully understood. We studied the effects of BCG infection (Connaught strain) on target cancer cells and host immune systems in murine bladder cancer. The bladder cancer cell line, MB49, was used in C57/BL6 mice in vivo and in vitro. In vitro cytotoxicities against the cancer cell line were measured by $24-\mathrm{h}{ }^{51} \mathrm{Cr}$ release assay. For effector cells, spleen mononuclear cells were obtained from mice injected intraperitoneally with BCG or BCG-infected irradiated MB49 cells. Although BCG infection of cancer cells did not affect the proliferation speed in vitro, the mice injected subcutaneously with BCG-infected MB49 cells survived significantly longer than those given untreated cancer cells. The mice surviving without tumor growth after injection of BCGinfected cancer cells could not reject a second injection of intact MB49 cells. In vitro cytotoxicity was enhanced by BCG infection of target cancer cells, but not by immunizing the mice with BCG from which effector cells were obtained. Moreover, cytotoxicity disappeared by depleting natural killer (NK) cells from effector cells. Although in vitro cytotoxicity was increased by immunizing the mice with BCG-infected irradiated MB49 cells, survival did not improve in these mice. These results suggest that a major part of BCG's anti-tumor effects can be attributed to the elimination of BCG-infected cancer cells by NK cells.
\end{abstract}

\section{Introduction}

Although superficial cancers of the urinary bladder can be mostly treated by transurethral resection (TUR) successfully,

Correspondence to: Dr Kazunobu Sugimura, Department of Urology, Osaka City University Graduate School of Medicine, 1-4-3 Asahi-machi, Abeno-ku, Osaka 545-8585, Japan

E-mail: ksugimura@msic.med.osaka-cu.ac.jp

Key words: bacillus Calmette-Guerin, bladder cancer, intravesical instillation, natural killer cells the post-TUR intravesical recurrence rate is $50-65 \%$. In order to prevent recurrence, intravesical instillation therapy has been tried with several anti-neoplasm drugs including anthracyclines and biological response modifiers, among which instillation of live Mycobacterium bovis or bacillus Calmette-Guerin (BCG) has resulted in the lowest recurrence rate $(1,2)$. In addition, intravesical BCG instillation is considered to be the most effective bladder-preservative treatment for carcinoma in situ (CIS) of the urinary bladder (3-5). In spite of these clinically favorable outcomes, the mechanisms by which BCG mediates anti-tumor activity have not been clearly understood, although immune mechanisms have been associated with the anti-tumor activity of BCG (6).

Various immunostimulatory effects of BCG in vitro and in vivo have been reported (7). It is generally accepted that BCG activates multiple cellular components such as $\mathrm{CD}^{+} \mathrm{T}$ cells, CD8 ${ }^{+} \mathrm{T}$ cells, NK cells and macrophages (8-11). In patients receiving intravesical BCG instillation therapy, a local inflammatory immune response characterized by an influx of mononuclear cells into the bladder wall and secretion of proinflammatory cytokines such as IL-1, IL-2, IL-6, tumor necrosis factor- $\alpha$ and interferon- $\gamma$ into the urine have been observed $(10,12,13)$. The cytokines are thought to augment the immune response, resulting in inhibition of tumor growth. From these studies, however, it is not clear which cell populations predominantly contribute to the anti-tumor activity of BCG. Furthermore, although the anti-tumor cytotoxic effect of BCG is undoubted, it remains unknown whether BCG actually has a prophylactic effect immunologically or whether its clinical prophylactic efficacy is just due to the eradication of cancer cells remaining after TUR.

In order to address these questions, we used an animal model to investigate the effects of BCG on cancer cells and on the host immune system, which enabled the evaluation of in vivo survival periods as well as in vitro cytotoxicities.

\section{Materials and methods}

Cell line. The murine bladder cancer cell line, MB49, originally induced by 7,12-di-methylbezabthrene, was kindly provided by Dr Timothy L. Ratliff. The cells were cultured in RPMI1640 medium containing $10 \%$ FCS and 100 units $/ \mathrm{ml}$ penicillin in a $5 \% \mathrm{CO}_{2}$ incubator at $37^{\circ} \mathrm{C}$. 
Animals. Male 8-week-old C57BL/6J mice were purchased from Nippon SLC (Shizuoka, Japan) 2 weeks before the experiments started. They were housed at maximally 5 mice/ cage and fed normal chow and tap water ad libitum in our animal facilities. The animal studies were approved by our Institutional Board.

Infection of cancer cells with BCG. Lyophilized BCG, Connaught strain (Immucyst, kindly provided by Nippon kayaku, Tokyo, Japan) containing $11.7 \times 10^{8}$ colony forming units (CFU)/81 mg dry weight, was used.

After $1 \times 10^{6}$ MB49 cancer cells were incubated overnight in $90-\mathrm{mm}$ diameter culture dishes at $37^{\circ} \mathrm{C}$ in a $\mathrm{CO}_{2}$ incubator, the culture medium was replaced with $10 \mathrm{ml}$ RPMI-1640 containing $10^{8} \mathrm{CFU}$ of BCG, $10 \%$ FCS and 100 units $/ \mathrm{ml}$ penicillin. After 7-day incubation, the cancer cells were collected by $0.05 \%$ trypsin and $0.02 \%$ EDTA treatment. Washed with saline twice, the infected cells were resuspended in RPMI-1640 and used for the experiments.

Measurement of cell proliferation. Premix WST-1 cell proliferation assay (Takara Biomedicals, Shiga, Japan) was performed to examine the influence of BCG infection on cancer cell proliferation. BCG-infected cells and control cells were seeded at $1 \times 10^{4}$ cells per well in 6-well tissue culture plates. After 48 -h incubation at $37^{\circ} \mathrm{C}, 100 \mu \mathrm{l}$ of WST-1 reagent was added to each well and allowed to react for $3 \mathrm{~h}$ at $37^{\circ} \mathrm{C}$, followed by measurement of absorbance at $450 \mathrm{~nm}$ using a microplate reader.

Immunization of mice. Each mouse was inoculated intraperitoneally with $1 \times 10^{7} \mathrm{CFU}$ of BCG two times at a 1 -week interval. In order to enhance specific anti-tumor immunities, we also immunized the mice with irradiated cancer cells. MB49 cells with or without BCG infection were irradiated with 25 Gy at $1.73 \mathrm{~Gy} / \mathrm{min}$ immediately before injection. BCG-infected cells were washed twice with saline to decrease the direct effects of BCG. Each mouse was inoculated intraperitoneally with $2 \times 10^{6}$ irradiated cells twice at a 1 -week interval. The control mice were injected with the medium only using the same schedule.

One week after the second injection, the immunized mice were used for either isolation of splenic mononuclear cells (MNCs) or survival experiments with MB49 injections.

In vivo survival experiments. The immunized mice were subcutaneously inoculated in the back with $200 \mu 1$ of a suspension containing $1 \times 10^{5}$ viable MB49 cells with or without BCG infection. Mice survival curves were calculated by the Kaplan-Meier method and compared by the log-rank test.

Isolation of splenic mononuclear cells (MNCs). The immunized mice were sacrificed and spleens removed aseptically. The spleens were crushed in culture medium, and the splenocytes were washed out by injecting the medium. To isolate the MNCs, the cells were separated by gradient centrifugation with Ficoll-Isopaque (density $=1.0875 \mathrm{~g} / \mathrm{cm}^{3}$ ) for $20 \mathrm{~min}$ at $1500 \mathrm{x}$ g. The interface was collected and subsequently washed two times in PBS. The MNCs were then adjusted to a concentration of $1 \times 10^{7}$ cells $/ \mathrm{ml}$ in the culture medium and used in the cytotoxicity assay.

Subset depletion from MNCs. Depletion of lymphocyte subsets from the MNCs was performed by antibody-coated magnetic beads using the BD IMag cell separation system (BD Biosciences, San Diego, USA) according to the manufacturer's recommendations. Anti-mouse CD4 particles-DM, antimouse CD8 particles-DM and anti-mouse CD49b/Pan-NK cells plus anti-PE particles 2-DM were used for depletion of $\mathrm{CD}^{+} \mathrm{T}$ cells, $\mathrm{CD} 8^{+} \mathrm{T}$ cells and $\mathrm{NK}$ cells, respectively. The appropriately-coated particles were added at $50 \mu 1$ for every $10^{7} \mathrm{MNCs}$ and refrigerated at $6-12^{\circ} \mathrm{C}$ for $30 \mathrm{~min}$. The binding cells were removed by placing the tubes in a rack with a magnet attached. The purity of the eluted fractions was $>95 \%$ as determined by flow cytometry.

Cytotoxicity assay. Cytotoxicities were measured by means of a 24-h chromium-release assay using MB49 cells or BCGinfected MB49 cells as target cells and splenic MNCs as effector cells. Target cells of $10^{6}$ were radiolabelled with $200 \mu \mathrm{Ci}$ of $\mathrm{Na}_{2}{ }^{51} \mathrm{CrO}_{4}$ (Amersham International, Amersham, Buckinghamshire, UK) at $37^{\circ} \mathrm{C}$ for $1 \mathrm{~h}$. After being washed twice, the labelled target cells were resuspended to $1 \times 10^{5}$ cells/ $\mathrm{ml}$ in the culture medium and were dispensed into 96-well U-bottomed microtitre plates at $1 \times 10^{4}$ cells/well. Effector cells were added to the appropriate wells to give effectortarget cell ratios of 100:1 in a total of $300 \mu 1$ of the culture medium. After $24-\mathrm{h}$ incubation at $37^{\circ} \mathrm{C}$ in a $5 \% \mathrm{CO}_{2}$ incubator, $100 \mu \mathrm{l}$ of the culture supernatants were obtained and counted in a gamma counter. Maximum isotope release was measured by incubation of the targets in $0.5 \mathrm{~N} \mathrm{NaOH}$. Spontaneous isotope release was measured by incubation of the targets in the culture medium. The following formula was used to calculate cytotoxicity: $\%$ specific cytotoxicity $=100 \mathrm{x}$ [experimental release $(\mathrm{cpm})$-spontaneous release $(\mathrm{cpm})] /[$ maximum release (cpm)-spontaneous release (cpm)]. Each assay was performed in triplicate.

Statistical analysis. All data were presented as means \pm SD. Student's t-test was used for comparison, and $\mathrm{p}<0.05$ was considered statistically significant.

\section{Results}

Influence of BCG infection on proliferation of MB49 cells. In order to study the direct effect of BCG infection on cancer cells (MB49), we determined the proliferation rate in vitro. BCG-infected MB49 cells proliferated at the same speed as intact cells (Fig. 1). Counting viable cell number by trypan blue staining also showed no difference by BCG infection (data not shown), confirming that BCG has no direct anti-tumor effects.

Survivals of mice inoculated with MB49 cells. The mice inoculated with BCG-infected MB49 cells survived significantly longer than those inoculated with untreated MB49 cells (Fig. 2). By the end of the observation period (60 days), all mice inoculated with intact MB49 cells had died, while $20 \%$ of the mice inoculated with BCG-infected MB49 cells were alive 


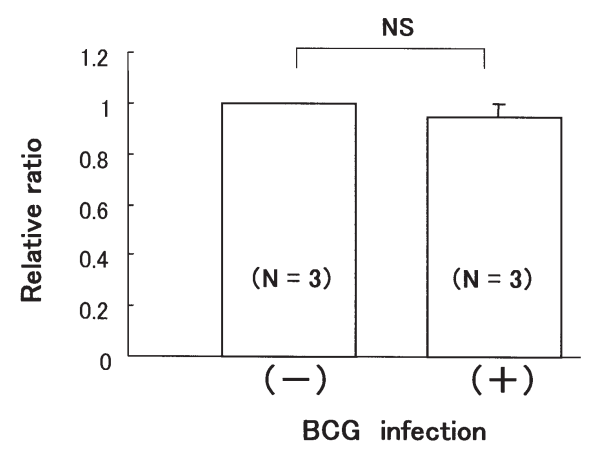

Figure 1. Influence of BCG infection on proliferation of MB49 cells.

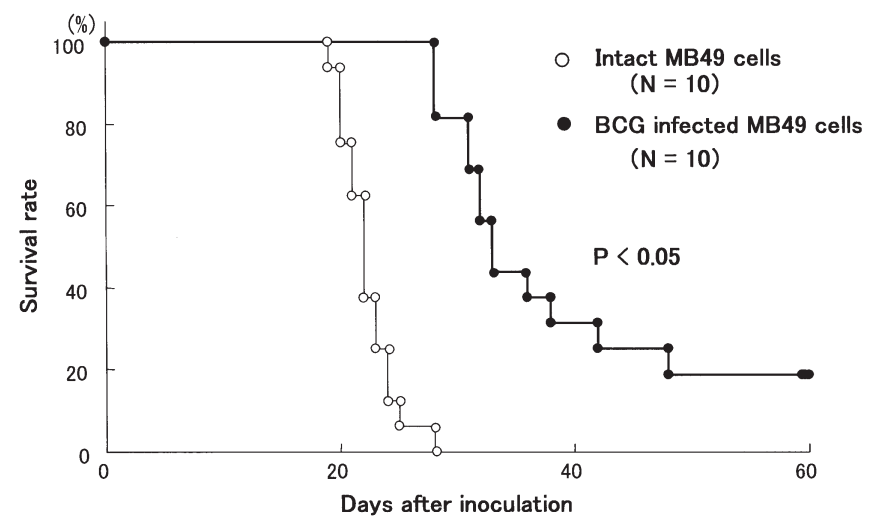

Figure 2. Survival of mice inoculated with MB49 cells. The mice were subcutaneously inoculated in the back with $200 \mu 1$ of suspension containing $1 \times 10^{5}$ viable MB49 cells

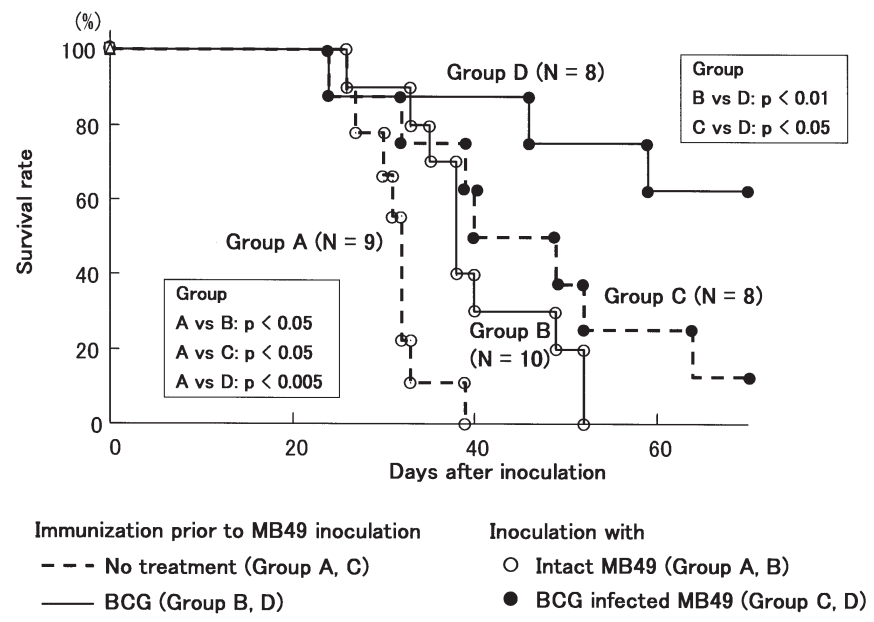

Figure 3. Survival of mice inoculated with MB49 cells after immunization with BCG. Each mouse was intraperitoneally injected with $1 \times 10^{7} \mathrm{CFU}$ of BCG two times at a 1-week interval. One week after the second injection, the mice were subcutaneously inoculated in the back with $200 \mu 1$ of suspension containing $1 \times 10^{5}$ viable MB49 cells.

without any growth of tumors, as the inoculated cells were most likely eliminated by the mouse immune systems.

We next examined the effects of immunization on survivals of the mice inoculated with MB49 cells. Prior to MB49 inoculation, the mice were immunized with BCG, irradiated

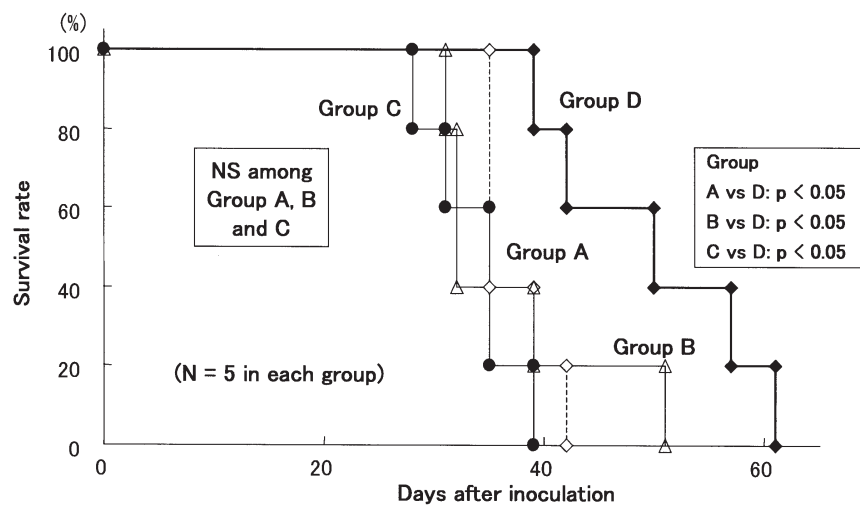

$$
\begin{aligned}
& \text { Pretreatments (immunization) prior to inoculation with } 1 \times 10^{5} \text { intact MB49 cells. } \\
& \text { Group A }-\quad \text { Medium (RPMI1640) } \\
& \text { B } \longrightarrow \quad \text { NMB49 (irradiated MB49 cells without BCG infection) } \\
& \text { C } \longrightarrow \text { B MB49 (irradiated MB49 cells with BCG infection) } \\
& \text { D } \longrightarrow \text { BCG }
\end{aligned}
$$

Figure 4. Survival of mice inoculated with MB49 after immunization with irradiated MB49 cells. Each mouse was intraperitoneally injected with $2 \times 10^{6}$ irradiated cancer cells with or without BCG infection two times at a 1-week interval. One week after the second injection, the mice were subcutaneously inoculated in the back with $200 \mu 1$ of suspension containing $1 \times 10^{5}$ viable MB49 cells.

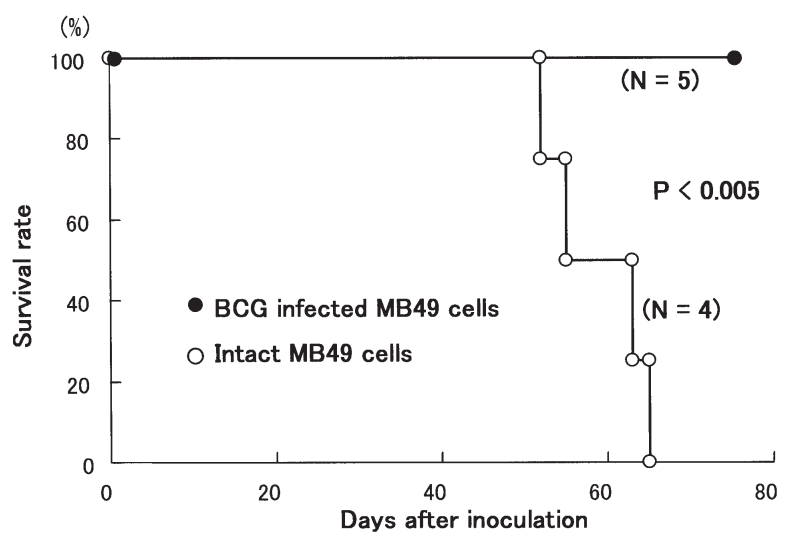

Figure 5. Rechallenge of surviving mice with MB49 cells. The mice that rejected the inoculated MB49 cells with BCG infection and survived at least 3 months without tumor growth were inoculated subcutaneously in the back with MB49 cells with or without BCG infection.

MB49 cells or irradiated MB49 cells infected with BCG. Immunization with intraperitoneal BCG injection resulted in a significantly longer survival both after intact MB49 and BCG-infected MB49 inoculation (Fig. 3). However, no significant effect was observed on survival by immunizing the mice with irradiated MB49 cells, and the survival curve was not improved by BCG infection of irradiated MB49 cells (Fig. 4).

Rechallenge of surviving mice with MB49 cells. The mice that rejected the inoculated BCG-infected MB49 cells and survived at least 3 months without tumor growth were injected subcutaneously in the back with $1 \times 10^{5}$ viable MB49 cells again with or without BCG infection. None of the 4 mice inoculated with untreated MB49 cells survived, while all 5 mice inoculated with BCG-infected MB49 cells survived again without any tumor growth (Fig. 5). 


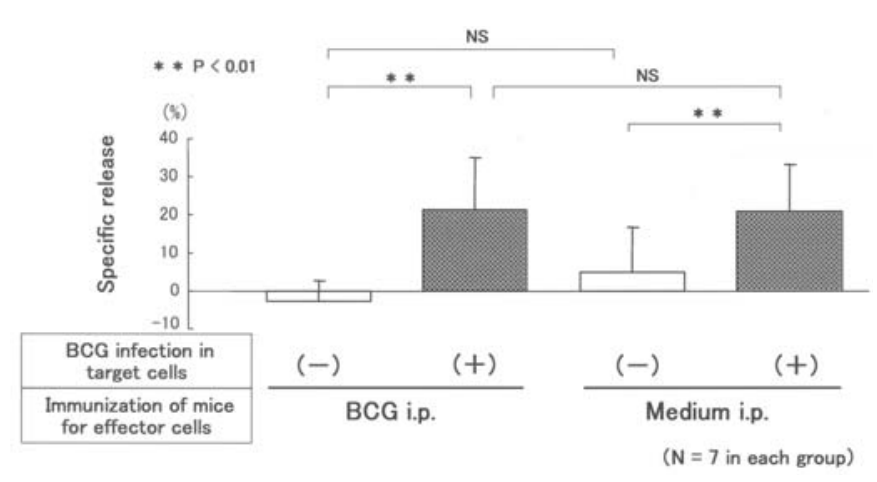

Figure 6 . The effects of BCG infection on in vitro cytotoxicity by ${ }^{51} \mathrm{Cr}$ release assay. Target cells were MB49 cells with or without BCG infection. For effector cells, the spleen mononuclear cells were isolated either from the mice immunized with BCG or the control mice injected with saline.

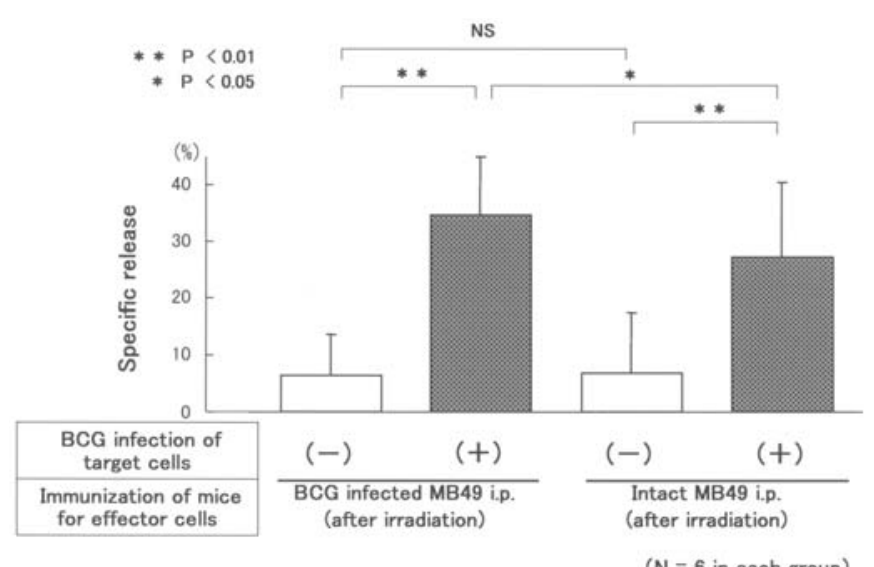

Figure 7. The effects of BCG infection on in vitro cytotoxicity by ${ }^{51} \mathrm{Cr}$ release assay. Target cells were MB49 cells with or without BCG infection. For effector cells, spleen mononuclear cells were isolated from the mice immunized with irradiated MB49 cells with or without BCG infection.

In vitro cytotoxicities against MB49 cells. The cytotoxicities against MB49 cells were determined by the 24-h chromiumrelease assay, and the influences of BCG infection on effector cells and target cells were examined separately. When target MB49 cells were infected with BCG, cytotoxicity was significantly greater than that in the absence of BCG infection (Fig. 6), which was consistent with the results of our in vivo survival experiments. On the other hand, cytotoxicity was not increased by BCG immunization of the mice from which the effector cells were obtained. Because the number of effector cells was constant in these in vitro assays, this result is not necessarily contrary to the survival improvement by BCG immunization shown in Fig. 3, if BCG treatment increases the number of cytotoxic MNCs without altering the ratio of subset populations.

Next, we examined the effects of BCG infection on cytotoxicity when effector cells were isolated from the mice immunized with irradiated MB49 cells (Fig. 7). The MNCs from the mice immunized with BCG-infected irradiated MB49 cells tended to show higher cytotoxicity than those from the mice immunized with untreated MB49 cells, with a statistical significance when target cells were infected with BCG.

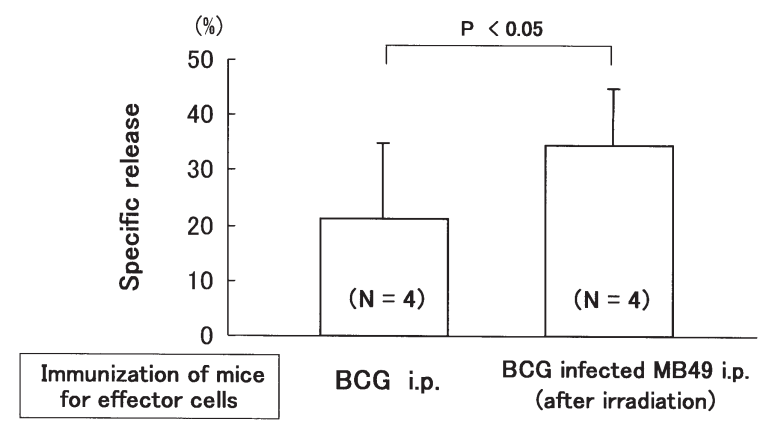

Figure 8 . In vitro cytotoxicity by ${ }^{51} \mathrm{Cr}$ release assay. Target cells were BCGinfected MB49 cells. Effector cells were spleen mononuclear cells isolated from the mice immunized with BCG or irradiated BCG-infected MB49 cells.

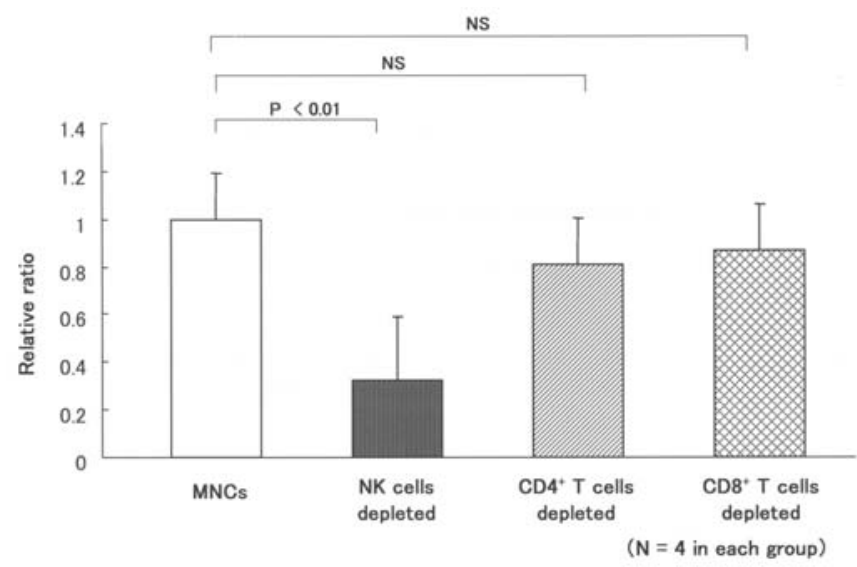

Figure 9. The effects of lymphocyte subset depletion on cytotoxicity against BCG-infected MB49 cells. For effector cells, spleen mononuclear cells (MNCs) were isolated from the BCG-immunized mice, and either NK cells, $\mathrm{CD}^{+} \mathrm{T}$ cells or $\mathrm{CD} 8^{+} \mathrm{T}$ cells were removed by antibody-coated magnetic beads.

Moreover, these MNCs from the mice immunized with BCGinfected MB49 cells showed greater cytotoxicity than those from the BCG-immunized mice (Fig. 8). This in vitro result is not consistent with survival results shown in Fig. 4, implying that tumor-specific immunity is not enough to improve survival periods at least in our animal model, even if specific immunity is induced by BCG-infected cancer cells.

The effects of lymphocyte subset depletion. In order to identify the subpopulation of MNCs mediating or being responsive for the cytotoxicities to BCG-infected MB49 cells, we removed cell subpopulations from the MNCs by magnetic beads. The MNCs from the BCG-immunized mice were depleted of CD4, CD8 and NK cells, respectively. As shown in Fig. 9, depletion of NK cells resulted in a significant $(\mathrm{P}<0.01)$ decrease of cytotoxicity, while depletion of CD4 or CD8 cells did not, demonstrating that non-specific NK cells are predominantly attributed to the cytotoxicity against BCGinfected cancer cells.

Cytotoxic subpopulations were also examined in the MNCs from the mice immunized with irradiated MB49 cells with BCG infection (Fig. 10). Depletion of either CD4 or CD8 


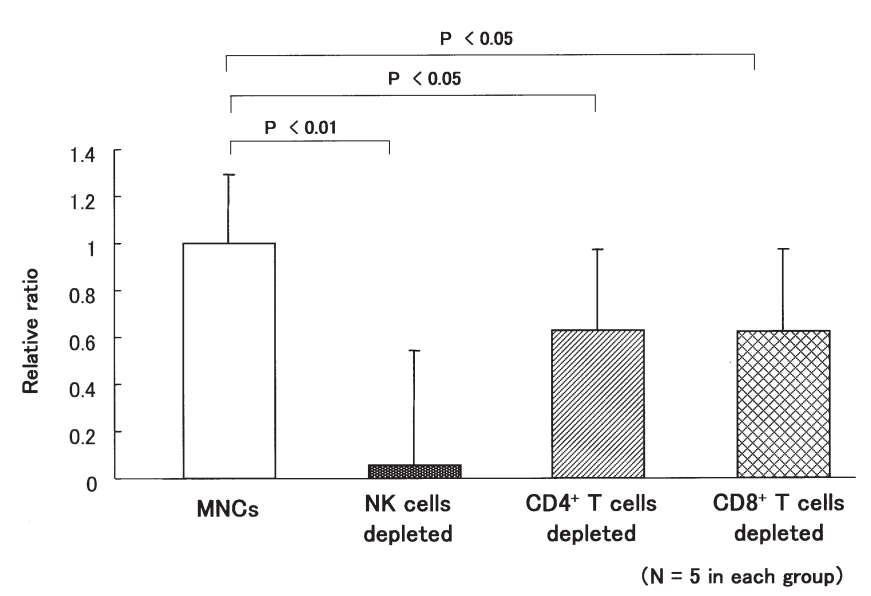

Figure 10. The effects of lymphocyte subset depletion on cytotoxicity against BCG-infected MB49 cells. For effector cells, spleen mononuclear cells (MNCs) were isolated from the mice immunized with BCG-infected irradiated MB49 cells, and either NK cells, CD4 ${ }^{+} \mathrm{T}$ cells or $\mathrm{CD}^{+} \mathrm{T}$ cells were removed by antibody-coated magnetic beads.

cells showed a significant decrease of cytotoxicity by one third, suggesting that specific immunity was induced by BCG-infected cancer cells. Nevertheless, depletion of NK cells resulted in a much greater decrease of cytotoxicity, so that the major cytotoxic subpopulation was revealed to be non-specific NK cells.

\section{Discussion}

To discuss the prevention of intravesical recurrence of urothelial cancers, the mechanisms for recurrence must be understood. There are two major hypotheses explaining the multifocality and recurrence of urothelial cancer; namely field defect of carcinogens resulting in de novo transformed cancer cells, and luminal seeding of the original clone $(14,15)$. Because multiple or recurrent tumors demonstrate oligoclonality or monoclonality respectively according to each hypothesis, the clonality of synchronous and metachronous urothelial tumors has been studied at the genomic level over the last decade. However, the results have been controversial, implying that both cases can occur in recurrences.

There is a hypothesis that acquired specific anti-tumor immunity plays an important role in post-TUR BCG therapy to prevent recurrence through memorizing tumor antigens (16). However, our result that the mice surviving after the first BCG-treated cancer inoculation could not reject a second inoculation without BCG infection, suggests that BCG therapy cannot enhance specific anti-tumor immunity enough to prevent recurrence, even if the recurrent tumors are the same clone of the origin. Therefore, its clinical effect preventing recurrence can be attributed to the strong cytotoxicity to kill cancer cells remaining after TUR whether they are monoclonal or oligoclonal.

Our result that immunization with irradiated MB49 cells with or without BCG infection did not improve the survival rates also suggests no significant contribution of specific antitumor immunity in this model, although our in vitro results clearly showed that cytotoxicity is increased by immunization with BCG-infected MB49 cells. One explanation for this contradiction is the difference in the sites of immunization and cancer inoculation. The survival study was performed by intraperitoneal immunization followed by subcutaneous inoculation in the back, while effector cells in the cytotoxicity assay were from the spleen, the same region of immunization. If regional lymph nodes play a crucial role in anti-tumor immunity, different injection locations may bring about different results.

On the other hand, BCG immunization resulted in longer survival both for untreated and BCG-infected MB49 inoculated mice. It is difficult to explain this effect by tumor-specific immunity, which is also supported by our finding that in vitro cytotoxicity was not increased by BCG pretreatment of effector cells. It is more likely that BCG immunization increased innate immunity mediated by NK cells, resulting in survival improvement. Clinically, intravesical BCG therapy is equally effective in countries like the United States where BCG is not used for prophylaxis of tuberculosis as well as in countries like Japan where BCG is used nationwide (17). Judging from this epidemiological finding, acquired immunity for BCG is not essential for this treatment, which is consistent with our results when explained by innate immunity.

One of the distinguished features of BCG therapy is that the cytotoxic effect is only against the cells infected with BCG, as our in vitro and in vivo studies also showed. In case of CIS of the bladder which is mostly treated by intravesical BCG instillation therapy successfully (18), most cancer cells can be infected with BCG by repeated intravesical instillation, resulting in a high anti-tumor efficacy. However, in animal models forming subcutaneous tumor masses, it seems impossible to infect all cancer cells with BCG after solid tumors are formed, resulting in no therapeutic effects of BCG. In the present study, we inoculated the cancer cells after co-incubating with BCG, by which the efficacy of BCG could be successfully demonstrated as survival periods, showing that this simple method is a useful animal model to investigate BCG therapy.

The other important phenomenon of BCG therapy is that only living BCG has anti-tumor effects. These two features are very understandable when the anti-tumor effects of BCG are considered to be based on the host immune system eliminating intracellular Mycobacterium. Although there is a possibility that BCG infection enhances specific anti-tumor immunity, it is more likely that the cells to be killed because of Mycobacterium infection are cancerous cells by chance. Concerning the cell-mediated immunity against intracellular parasitic Mycobacterium, it is known that activated macrophages play an important role $(19,20)$. However, our in vitro study depleting lymphocyte subsets clearly showed the significance of NK cells in this BCG model.

In conclusion, we were able to demonstrate the anti-tumor effects of BCG as survival periods by BCG infection before cancer cell inoculation. In this model, a major part of BCG anti-tumor effects could be attributed to the elimination of BCG-infected cancer cells by NK cells rather than the enhancement of specific anti-tumor immunity.

\section{Acknowledgements}

This study was supported by the Osaka City University Medical Research Foundation. 


\section{References}

1. Lamm DL, Riggs DR, Traynelis CL and Nseyo UO: Apparent failure of current intravesical chemotherapy prophylaxis to influence the long-term course of superficial transitional cell carcinoma of the bladder. J Urol 153: 1444-1450, 1995.

2. Pawinski A, Sylvester R, Kurth KH, Bouffioux C, van der Meijden A, Parmar MK and Bijnens L: A combined analysis of European Organization for Research and Treatment of Cancer, and Medical Research Council randomized clinical trials for the prophylactic treatment of stage TaT1 bladder cancer. European Organization for Research and Treatment of Cancer Genitourinary Tract Cancer Cooperative Group and the Medical Research Council Working Party on Superficial Bladder Cancer. J Urol 156: 1934-1940, 1996.

3. Morales A, Eidinger D and Bruce AW: Intracavitary bacillus Calmette-Guerin in the treatment of superficial bladder tumors. J Urol 116: 180-183, 1976 .

4. Smith JA Jr, Labasky RF, Cockett AT, Fracchia JA, Montie JE and Rowland RG: Bladder cancer clinical guidelines panel summary report on the management of non-muscle invasive bladder cancer (stages Ta, T1 and TIS). J Urol 162: 1697-1701, 1999.

5. Herr HW, Schwalb DM, Zhang ZF, Sogani PC, Fair WR, Whitmore WF Jr and Oettgen HF: Intravesical bacillus CalmetteGuerin therapy prevents tumor progression and death from superficial bladder cancer: ten-year follow-up of a prospective randomized trial. J Clin Oncol 13: 1404-1408, 1995.

6. Ratliff TL: Role of the immune response in BCG for bladder cancer. Eur Urol 21 (suppl 2): S17-S21, 1992.

7. Alexandroff AB, Jackson AM, O'Donnell MA and James K: BCG immunotherapy of bladder cancer: 20 years on. Lancet 353: 1689-1694, 1999.

8. Rubenstein M, Muchnik S, Chet M, Shaw MW, McKiel CF and Guinan PD: Tumor infiltrating lymphocytes: the effect of bacillus Calmette Guerin on helper/suppressor-T cell ratios of treated and untreated tumors. J Urol 146: 1650-1653, 1991.

9. Leong AS, Wannakrairot P, Jose J and Milios J: Bacillus CalmetteGuerin-treated superficial bladder cancer: correlation of morphology with immunophenotyping. J Pathol 162: 35-41, 1990.
10. Bohle A, Gerdes J, Ulmer AJ, Hofstetter AG and Flad HD Effects of local bacillus Calmette-Guerin therapy in patients with bladder carcinoma on immunocompetent cells of the bladder wall. J Urol 144: 53-58, 1990.

11. El-Demiry MI, Smith G, Ritchie AW, James K, Cumming JA, Hargreave TB and Chisholm GD: Local immune responses after intravesical BCG treatment for carcinoma in situ. Br J Urol 60: 543-548, 1987.

12. Fleischmann JD, Toossi Z, Ellner JJ, Wentworth DB, Ratliff TL and Imbembo AL: Urinary interleukins in patients receiving intravesical bacillus Calmette-Guerin therapy for superficial bladder cancer. Cancer 64: 1447-1454, 1989.

13. Prescott S, James K, Hargreave TB, Chisholm GD and Smyth JF: Radio-immunoassay detection of interferon-gamma in urine after intravesical Evans BCG therapy. J Urol 144: 1248-1251, 1990.

14. Sidransky D, Frost P, von Eschenbach A, Oyasu R, Preisinger AC and Vogelstein B: Clonal origin bladder cancer. N Engl J Med 326: 737-740, 1992 .

15. Jones TD, Wang M, Eble JN, et al: Molecular evidence supporting field effect in urothelial carcinogenesis. Clin Cancer Res 11: 6512-6519, 2005

16. Reichert DF and Lamm DL: Long term protection in bladder cancer following intralesional immunotherapy. J Urol 132: 570-573, 1984.

17. Davis JW, Sheth SI, Doviak MJ and Schellhammer PF: Superficial bladder carcinoma treated with bacillus Calmette-Guerin: progression-free and disease specific survival with minimum 10-year followup. J Urol 167: 494-500, 2002.

18. Hudson MA and Herr HW: Carcinoma in situ of the bladder. J Urol 153: 564-572, 1995.

19. Van Furth R: Mycobacteria and macrophage activation. Res Microbiol 141: 256-261, 1990.

20. Dannenberg AM Jr: Roles of cytotoxic delayed-type hypersensitivity and macrophage-activating cell-mediated immunity in the pathogenesis of tuberculosis. Immunobiology 191: 461-473, 1994. 